\title{
Group theory Character Table Enhancement by Introducing the Partial Molecular Symmetry Principle in the Molecular Spectroscopy
}

\author{
Ahmed S. Abdel-Rahman \\ Physics Department, Faculty of Science, Cairo University, Giza, Egypt. \\ Corresponding author: asabry@sci.cu.edu.eg
}

Group theory character table is a very useful tool to know important information about molecular activities and their spectra but suffers different defects such as the physical meaning of $\omega$, the entire levels of degenerate states and the atomic motion through molecular vibration. On the way to define the infrared spectra, Raman spectra and natural frequency of molecules; it is important to split degenerate states and determine the motion of each atom through molecular vibration. While symmetry can be seen as the most basic and important concept in physics, the higher $n$-fold rotation axis $C_{n}$ symmetry element can be represented as a full or partial symmetric rotation axis and governs other symmetry elements in the molecule through the vibrational motion. Symmetry states and Omega-table which hold all possible symmetry states of the molecule will solve the character table defects. Selection rules judge some of the symmetry states to be forbidden. Omega-table illustrates the relation between symmetry elements in the molecule through its translation, rotation and vibration processes and separates the degenerate states as well as can be reduced to point group character table. Based on omegatable, the description of the atomic motion of $\mathrm{NH}_{3}$ molecule or any other molecules through vibration was so clear according to $\omega$ mathematical value and its physical meaning.

\section{Introduction:}

Molecules can be classified into point groups according to their symmetry elements. Molecules with similar symmetries are put into the same point group. Symmetry elements are mirror planes, axes of rotation (n-fold rotation axis), centers of inversion, etc.

On a given physical system, the eigenstate properties and the degeneracy of eigenvalues are governed by symmetry considerations. The symmetry properties 
of each point group are summarized in a character table. Character table lists all symmetry elements of the group, along with a complete set of irreducible representations [1].

The point group character table is useful to investigate the polarity, chirality, orbitals used in $\sigma$ bonds and symmetry properties of all molecular motions in molecules [1-4]. But suffers some defects in its construction, and they are:

- The physical and mathematical meaning of $\omega$,

- Combined symmetry elements,

- Degenerate states,

- Meaning of characters rather than \pm 1 .

The character $\omega$ was very important as predicted by F. Hegelund et al. [5], it is the $n$-th root of unity and being a complex quantity:

$$
\omega_{n}=e^{2 \pi i / 3}
$$

Application of character table leads to define the infrared and Raman spectra relation to the different vibration modes, but cannot determine the spectrum frequency and intensity where character table fails to describe the atomic motion through molecular vibration. The application of character table and finding the corresponding spectral line in IR and Raman studies are investigated on many molecules [6-8].

In the present work, the physical definition of $\omega$ will be investigated besides the building of omega-table as the general case of character table, which solves character table defects and will show the vibration motion of each atom in ammonia molecule as the $C_{3 v}$ example in contrast with what is illustrated in $[1,2]$.

\section{Defects in the Character Table:}

Table 1 shows the character table of the $C_{3}$ point group.

Table (1): Character table for the group $\mathrm{C}_{3}$.

\begin{tabular}{|c|c|c|c|}
\hline & $E$ & $C_{3}$ & $C_{3}^{2}$ \\
\hline$A$ & 1 & 1 & 1 \\
\hline \multirow{2}{*}{$E\{$} & 1 & $\Omega$ & $\omega^{2}$ \\
\cline { 2 - 4 } & 1 & $\omega^{2}$ & $\omega$ \\
\hline
\end{tabular}

\subsection{Physical and Mathematical Meaning of $\omega$}

Some characters as in Table (1), take complex values $(\omega)$, its physical and mathematical meanings are not so clear. The mathematical meaning is not that its value but what is meant by character to be a complex quantity. 


\subsection{Combined Symmetry Elements}

Some symmetry elements in character tables are combined in one column (such as $2 \mathrm{C}_{3}$ in the $C_{3 v}$ point group Table 2) and their characters represent collected values of its symmetry elements. The character table cannot illustrate each symmetry element value and then its case in molecular motions.

Table (2): Character table for group $\mathrm{C}_{3 \mathrm{v}}$.

\begin{tabular}{|c|c|c|c|}
\hline & $E$ & $2 C_{3}$ & $3 \sigma_{v}$ \\
\hline$A_{1}$ & 1 & 1 & 1 \\
\hline$A_{2}$ & 1 & 1 & -1 \\
\hline$E$ & 2 & -1 & 0 \\
\hline
\end{tabular}

\subsection{Degenerate States:}

Some irreducible representations displayed in double or triple or higher degenerate states, $E$ double degenerate state in the $C_{3 v}$ table 2, they do not show characters of the entire states.

\subsection{Meaning of characters rather than \pm 1}

It is well known, Characters of +1 indicate that the basic function is unchanged by the symmetry operation. While characters of -1 indicate that the basic function is reversed. Another character indicates that the basis function undergoes a more complicated change.

\section{Method}

\subsection{Symmetry elements in molecular motion}

The symmetry elements such as mirror planes, axes of rotation, centers of inversion are defined clearly for static molecules $[3,4]$. The molecular motions (translation, rotation and vibration) will show new symmetry modes rather than static due to the mutual motion of atoms in the molecule. The three-fold axis in the $\mathrm{C}_{3 \mathrm{v}}$ point group (ammonia as an example) can be discussed in three classes. The normal full symmetry axis $\mathrm{C}_{3}$ is shown in Fig. (1. a), where all atoms move in-phase. If one atom gets out from in-phase motion, the $\mathrm{C}_{3}$ symmetry element is not completely saved but shows a mid-way class between full symmetric and asymmetric classes. And then displays a new order of symmetry, this is called partial symmetry state and shown in Fig. (1. b). It is important to consider the motion of Nitrogen atom to conserve the center of mass in front of the Hydrogen plan (the plan of the paper). The two in-phase motions (two hydrogen atoms) should have vibration paths to conserve the angle of the $C_{3}$ axis on $120^{\circ}$. 


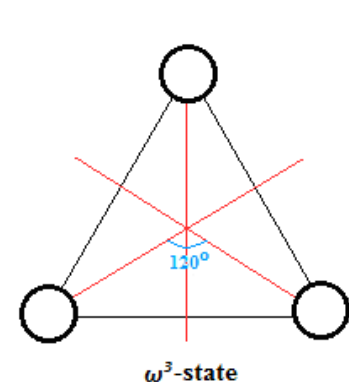

a

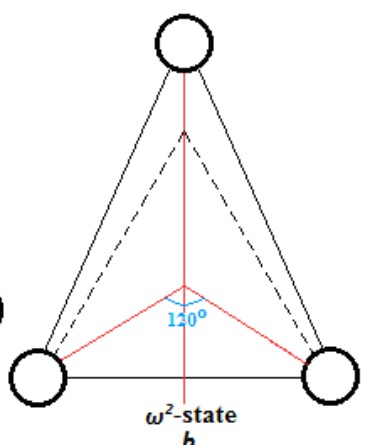

$b$

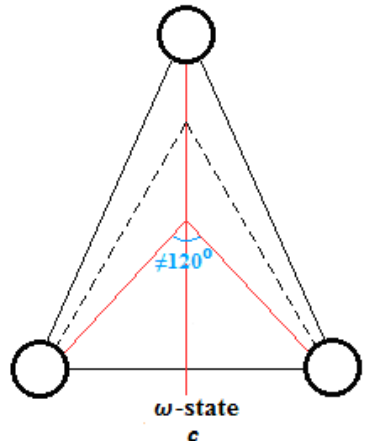

Fig. (1): The $\mathrm{C}_{3}$ fold axis states in molecular motion. (a) Full symmetry state, (b) partial symmetry state and (c) asymmetry state.

But it is forbidden for the three atoms to lose their in-phase motion totally while they must show a little amount of symmetry such as breaking the $\mathrm{C}_{3}$ angle condition only and keeping the two atoms on the same distance from rotation axis. Thus the $\mathrm{C}_{3}$ axis disappears and this case is denoted by asymmetry state (Fig. 1. c).

Based on this hypothesis, the new concept can be defined as the n-fold axis has $n$ symmetry states (these states are only applicable in the case of molecular vibration or generally in molecular motion). Two-fold rotation axis (or mirror plane and center of inversion) has two allowed states. First is the symmetric state while the molecule coincides itself through $180^{\circ}$ rotation and other is the asymmetric state. The identity $E$ is valid anyway and takes single state (symmetric state).

\subsection{Omega Definition:}

All symmetry elements in the molecule will be denoted mathematically by $\omega_{n}{ }^{m}$, while $n$ is the index of omega and takes values $1,2, \ldots, 6 ; m$ is the order of omega and takes the values $1,2, \ldots, n$ where omega $\omega$ is the $n^{\text {th }}$ root of unity (Eq. 1). Mirror planes and center of inversion have the index $n=2$, while the $\mathrm{n}$-fold axis has the index of $n$. The order of omega defines the symmetry ratio to the full symmetry state or in other words, $m=n$ is the full symmetric and $m=1$ is the asymmetric state.

\subsection{Symmetry States:}

As the molecular motions happen, all permutations of symmetry states are considered to form molecular symmetry states. Each symmetry element is labeled in a separate column while the rows will hold all possible permutations of omega values. Table (3) represents the molecular symmetry states for the $C_{3 v}$ point group and three mirror planes in the direction of the major three-fold rotation axis $\mathrm{C}_{3}$, the $\mathrm{C}_{3}{ }^{2}$ axis is the rotation axis as $\mathrm{C}_{3}$ in the opposite rotation direction. 
Table 3: $\mathrm{C}_{3 \mathrm{v}}$ symmetry States.

\begin{tabular}{|c|c|c|c|c|c|}
\hline $\mathbf{E}$ & $\mathrm{C}_{3}(\mathrm{z})$ & $\mathrm{C}_{3}^{2}(\mathrm{z})$ & $\sigma_{\mathrm{v}}(\mathbf{x z})$ & $\sigma_{\mathrm{v} 2 \mathrm{z}}$ & $\sigma_{\mathrm{v} 3 z}$ \\
\hline$\omega_{1}$ & $\omega_{3}^{3} / \omega_{3}^{2} / \omega_{3}$ & $\omega_{3}^{3} / \omega_{3}^{2} / \omega_{3}$ & $\omega_{2}^{2} / \omega_{2}$ & $\omega_{2}^{2} / \omega_{2}$ & $\omega_{2}^{2} / \omega_{2}$ \\
\hline & \multicolumn{5}{|l|}{$\omega_{1}=e^{i 2 \pi / 1}=1$} \\
\hline
\end{tabular}

Not of all these symmetry states are allowed for the molecule to hold, some of these states are forbidden and they can be determined by selection rules.

\subsection{Selection Rules:}

All permutations of omega values in the $C_{3 \mathrm{v}} \omega$-table are 72 symmetry states; the main symmetry rotation axis judges the other symmetry elements' values throughout selection rules as following:

1- The opposite direction z-fold rotation axis $\left(\mathrm{C}_{3}{ }^{2}\right)$ is, in reality, is the $\mathrm{C}_{3}$ twice and then it must be equal to the square value of $\mathrm{C}_{3} \omega$-value. So if any symmetry state has $\mathrm{C}_{3}=\omega_{3}{ }^{\mathrm{m}}$ and $\mathrm{C}_{3}{ }^{2} \neq \omega_{3}{ }^{2 \mathrm{~m}}$, this state will be forbidden.

2- When the $\mathrm{C}_{3}$ is in $\omega_{3}{ }^{3}$-state (full symmetry) that defines two cases for lower elements (i.e. $3 \sigma_{v}$ ). In the first case, all of these planes are symmetric, and the other case all of them are anti-symmetric, then this state is being degenerate to two mutual states as above $A_{1}$ and $A_{2}$ while $A_{1}$ the symmetric state called $\omega_{a}{ }^{3}$-state and $\mathrm{A}_{2}$ the symmetric/asymmetric state called $\omega_{b}{ }^{3}$-state.

3- If the $\mathrm{C}_{3}$ is in $\omega_{3}$-state (asymmetric state) that defines at least one symmetric state of lower elements $\left(3 \sigma_{v}\right)$; this is the asymmetric state called $\omega$-state.

4- If the $\mathrm{C}_{3}$ in $\omega_{3}{ }^{2}$-state (partial symmetric state) this state requires one symmetric of the lower elements; this is the semi-symmetric/semi-symmetric state called $\omega^{2}$-state.

5- The application of selection rules on the $\mathrm{C}_{3 \mathrm{v}} \omega$-table reduces states from 72 to 4 allowed states as shown in Table (4). Table (5) illustrates the allowed states with their omega-equivalent values.

Table (4): The $\mathrm{C}_{3 \mathrm{v}} \omega$-table with all allowed/forbidden states.

\begin{tabular}{|c|c|c|c|c|c|c|c|}
\hline & $\mathbf{E}$ & $\mathbf{C}_{\mathbf{3}}(\mathbf{z})$ & $\mathbf{C}_{\mathbf{3}}{ }^{2}(\mathbf{z})$ & $\boldsymbol{\sigma}_{\mathbf{v}}(\mathbf{x z})$ & $\boldsymbol{\sigma}_{\mathbf{v} 2 \mathbf{z}}$ & $\boldsymbol{\sigma}_{\mathbf{v} 3 \mathbf{z}}$ & State \\
\hline $\mathbf{A}_{\mathbf{1}}$ & $\omega_{1}$ & $\omega_{3}{ }^{3}$ & $\omega_{3}{ }^{3}$ & $\omega_{2}{ }^{2}$ & $\omega_{2}{ }^{2}$ & $\omega_{2}{ }^{2}$ & allowed \\
\hline $\mathbf{A}_{\mathbf{2}}$ & $\omega_{1}$ & $\omega_{3}{ }^{3}$ & $\omega_{3}{ }^{3}$ & $\omega_{2}$ & $\omega_{2}$ & $\omega_{2}$ & allowed \\
\hline $\mathbf{E}_{\mathbf{1}}$ & $\omega_{1}$ & $\omega_{3}{ }^{2}$ & $\omega_{3}$ & $\omega_{2}{ }^{2}$ & $\omega_{2}$ & $\omega_{2}$ & allowed \\
\hline $\mathbf{E}_{\mathbf{2}}$ & $\omega_{1}$ & $\omega_{3}$ & $\omega_{3}{ }^{2}$ & $\omega_{2}{ }^{2}$ & $\omega_{2}$ & $\omega_{2}$ & allowed \\
\hline & \multicolumn{8}{|c|}{ Else } & forbidden \\
\hline
\end{tabular}


Table 5: The $\mathrm{C}_{3 \mathrm{v}}$ detailed $\omega$-table.

\begin{tabular}{|c|c|c|c|c|c|c|}
\hline & $\mathbf{E}$ & $\boldsymbol{\sigma}_{\mathbf{v}}(\mathbf{x z})$ & $\boldsymbol{\sigma}_{\mathbf{v}}(\mathbf{y z 1})$ & $\boldsymbol{\sigma}_{\mathbf{v}}(\mathbf{y z 2})$ & $\mathbf{C}_{\mathbf{3}}(\mathbf{z})$ & $\left.\mathbf{C}_{\mathbf{3}}{ }^{2} \mathbf{z}\right)$ \\
\hline $\mathbf{A}_{\mathbf{1}}$ & 1 & 1 & 1 & 1 & 1 & 1 \\
\hline $\mathbf{A}_{\mathbf{2}}$ & 1 & -1 & -1 & -1 & 1 & 1 \\
\hline $\mathbf{E}_{\mathbf{1}}$ & 1 & 1 & -1 & -1 & $\omega_{3}{ }^{2}$ & $\omega_{3}$ \\
\hline $\mathbf{E}_{\mathbf{2}}$ & 1 & 1 & -1 & -1 & $\omega_{3}$ & $\omega_{3}{ }^{2}$ \\
\hline
\end{tabular}

\section{Results and Discussion:}

\subsection{Reducing Omega-table to Character Table:}

Omega-table (Table 5) can be reduced to the well-known character table by merging the same type elements (columns) and degenerate states (rows) as following:

1- While the value of the $\mathrm{C}_{3}{ }^{2}$ axis just directly obtained from $\mathrm{C}_{3}$ axis values, one can merge these two elements in one element $\left(2 \mathrm{C}_{3}\right)$ and only mention the value of the $\mathrm{C}_{3}$ axis.

2- The independent elements $\sigma_{\mathrm{v}}(\mathrm{xz}), \sigma_{\mathrm{v}}(\mathrm{yz} 1)$ and $\sigma_{\mathrm{v}}(\mathrm{yz} 2)$ can be merged to one column $\left(3 \sigma_{\mathrm{v}}\right)$ by numerically adding uncommon values, where if there duplicated values are added the second value will be dropped; thus the result of combining the $A_{1}$ state is summing of three minus ones, the second and third minus ones will be dropped and the resultant is -1 .

To get the character table (Table 2), one should represent real (nonimagery) values, where $\omega_{3}$ and $\omega_{3}{ }^{2}$ should be disappeared. The two degenerate states $E_{1}$ and $E_{2}$ should be merged in one row called double degenerate state $E$, the same procedure in step 2 is applied to columns rather than identity while this column values summed in the regular method. Taking into account the imagery omega values will be disappeared where $\omega_{3}{ }^{2}+\omega_{3}=-1$.

In this methodology, we can say omega-table is the generalized form of the character table and can be applied to describe information (like atomic vibration) that cannot be deduced from the character table.

\subsection{Vibration Modes in Ammonia:}

Character table can describe the vibration modes of ammonia by finding the reducible representation $\Gamma_{3 \mathrm{~N}}$ and reduce it to irreducible representations $[1,9,10]$.

Decomposition theory can be applied to Table (2) as: 


$$
\begin{aligned}
& \chi_{A 1}\left(\Gamma_{3 N}\right)=\frac{1}{6}[12+0+(3 \times 2)]=3 \\
& \chi_{A 2}\left(\Gamma_{3 N}\right)=\frac{1}{6}[12+0-(3 \times 2)]=1 \\
& \chi_{E}\left(\Gamma_{3 N}\right)=\frac{1}{6}[24+0+0]=4 \\
& \Gamma_{3 \mathrm{~N}}=3 \mathrm{~A}_{1}+\mathrm{A}_{2}+4 \mathrm{E} \\
& \Gamma_{\text {trans }}=\mathrm{A}_{1}+\mathrm{E} \\
& \Gamma_{\text {rot }}=\mathrm{A}_{2}+\mathrm{E} \\
& \Gamma_{\mathrm{vib}}=2 \mathrm{~A}_{1}+2 \mathrm{E}
\end{aligned}
$$

while $\mathrm{E}=\mathrm{E}_{1}+\mathrm{E}_{2}$,

$$
\Gamma_{\mathrm{vib}}=2 \mathrm{~A}_{1}+2 \mathrm{E}_{1}+2 \mathrm{E}_{2}
$$

\subsection{Stretching Vibration and Atomic Motion}

By the same algorithm of decomposition, stretching and bending modes can be found; considering the stretching vibration modes:

$$
\Gamma_{\text {stretches }}=\mathrm{A}_{1}+\mathrm{E}_{1}+\mathrm{E}_{2}
$$

Stretching vibration mode is associated with the motion of atoms along with their bonds between them; but also creating change in the angle between bonds. The simplest stretching case for ammonia is that all hydrogen atoms move directly towards N-atom along their N-H bonds, and then nitrogen atom should move perpendicular to $\mathrm{H}$ atoms plane towards the center $c$ to reserve center of mass (Figs 2 and 3) and thus the angles between bonds will be changed.

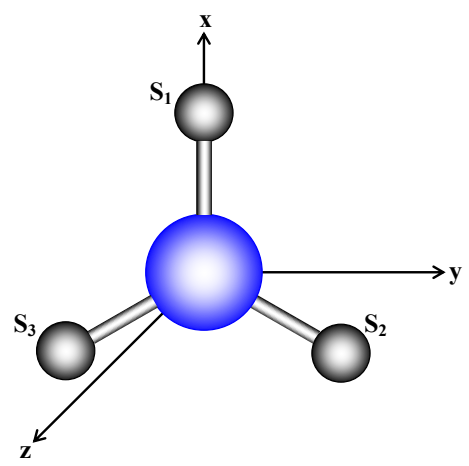

Fig. (2): Stretching vibration in Ammonia. 


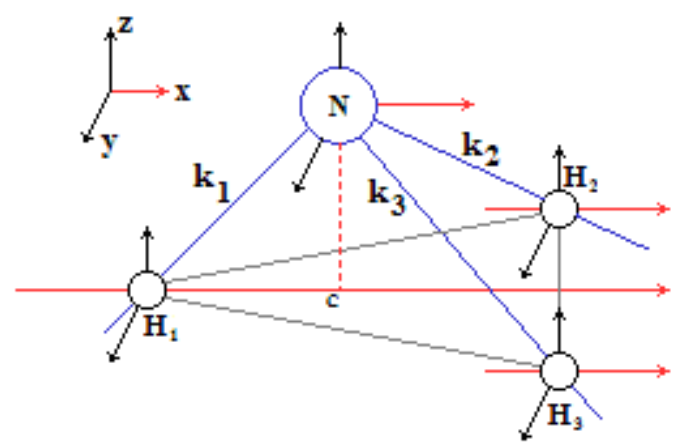

Fig. (3): Stretching vectors in Ammonia.

The reducible representation $\left(\chi_{\mathrm{Al}}\left(\mathrm{Q}_{\mathrm{S} 1}\right)\right)$ for the stretching mode $\mathrm{A}_{1}$ from the $\mathrm{S}_{1}$ atom is:

$$
\chi_{A 1}\left(Q_{S 1}\right)=\frac{1}{6}\left[S_{1}+S_{2}+S_{3}+S_{1}+S_{2}+S_{3}\right]=\frac{1}{3}\left[S_{1}+S_{2}+S_{3}\right]
$$

This represents a simple symmetric stretching mode $A_{1}$, where all atoms move in regular motion with constant amplitude along with their bonds.

$$
\begin{aligned}
& \chi_{E 1}\left(Q_{S 1}\right)=\frac{1}{6}\left[S_{1}+\omega_{3}^{2} S_{2}+\omega_{3} S_{3}+S_{1}-S_{2}-S_{3}\right]=\frac{1}{6}\left[2 S_{1}+\left(\omega_{3}^{2}-1\right) S_{2}+\left(\omega_{3}-1\right) S_{3}\right] \\
& =\frac{1}{6}\left[2 S_{1}+\left(-\frac{3}{2}-i \frac{\sqrt{3}}{2}\right) S_{2}+\left(-\frac{3}{2}+i \frac{\sqrt{3}}{2}\right) S_{3}\right]
\end{aligned}
$$

This vibration mode $E_{1}$ formed of moving $S_{1}$ atom $1 / 3$ points in $+x$ direction (along bond; i.e. $\mathbf{k}_{1}$-vector in Fig. 3) while $S_{2}$ atom moves $1 / 4$ points in $\mathrm{x}$-direction $\left(\mathbf{k}_{\mathbf{2}}\right)$ and $\sqrt{3} / 12$ points in $-\mathrm{y}$-direction (perpendicular to the bond $\mathbf{k}_{\mathbf{2}^{-}}$ vector), $S_{3}$ atom moves $1 / 4$ points in $-x$-direction $\left(\mathbf{k}_{3}\right)$ and $\sqrt{3} / 12$ points in $+y$ direction (perpendicular to the bond $\mathbf{k}_{3}$-vector).

To get the equation of motion of each atom, we choose the axis $\mathrm{x}$ to be set in the direction of an active mirror plane $\left(\sigma_{\mathrm{v}} \mathrm{xz}\right.$ in Table 5). $\mathbf{k}$ vector is the stretching vector of each bond toward the $\mathrm{N}$ atom.

Taking the projection of the molecule on xy-plan (the plan holds all hydrogen atoms) and nitrogen atom in front of the page as shown in Fig. (4) while the black circles define the static location of Hydrogen atoms and denoted by $\mathrm{H}$ while the gray circles define the atom location at maximum amplitude and denoted by H'. 


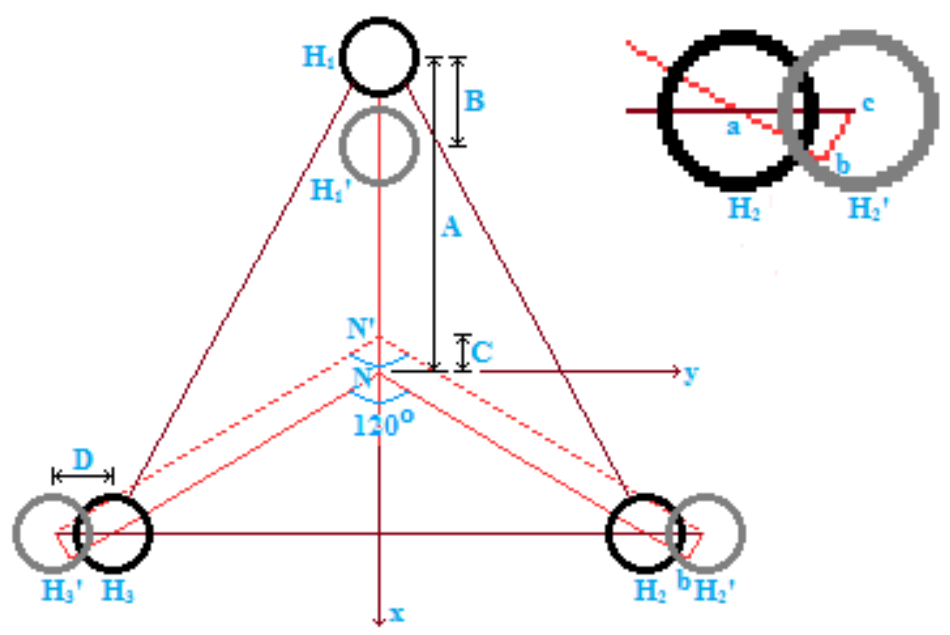

Fig. (4): Ammonia molecule projection on xy-plan.

The motion of $\mathrm{H}_{2}$ is $-3 / 2$-iv $3 / 2$ or in other words $3 / 2$ in the direction of $\mathbf{k}_{2}$ vector (projection is $\mathbf{a b}$ ) and $\sqrt{ } 3 / 2$ in the perpendicular direction (projection is bc), it is clear that the motion of $\mathrm{H}_{2}$ and $\mathrm{H}_{3}$ atoms lie in the y-direction outwards to each other to keep the angle to the $\mathrm{N}$-atom $\left(\mathrm{N}^{\prime}\right) 120^{\circ}$ through vibration and hence partial symmetry of $\mathrm{C}_{3}$ axis was saved. While the atoms $\mathrm{H}_{1}, \mathrm{H}_{2}, \mathrm{H}_{3}$, and $\mathrm{N}$ have their vibration amplitudes (xy-plane projection) as $B, D, D$, and $C$, respectively and they share the same frequency of vibration. And $A$ represents the $\mathrm{NH}$ bond projection on $\mathbf{x y}$-plane.

The stretching equation (6) describe the motion of atoms; $\mathrm{H}_{1}$ moves stretching towards $\mathrm{N}$ atom, while $\mathrm{H}_{2}$ and $\mathrm{H}_{3}$ move to expand each other, the $\mathrm{x}$ component of $\mathrm{H}_{2}$ ' can be calculated by two methods:

1) The motion of $\mathrm{H}_{2}{ }^{\prime}$ atom is being in the $y$-direction and hence the $\mathrm{x}$-component should be same as $\mathrm{H}_{2}$ atom, or $\mathrm{x}\left[\mathrm{H}_{2}{ }^{\prime}\right]=\mathrm{x}\left[\mathrm{H}_{2}\right]=\mathrm{A} \sin 60=\mathrm{A} / 2$

2) $\mathrm{x}\left[\mathrm{H}_{2}{ }^{\prime}\right]=\frac{1}{2}\left[\mathrm{~N}^{\prime} \mathrm{H}_{2}{ }^{\prime}\right]-C=\frac{1}{2}\left[\frac{C}{2}+A+\frac{\sqrt{3}}{2} D\right]-C=-\frac{3}{4} C+\frac{1}{2} A+\frac{\sqrt{3}}{4} D$

Now, these values must be equal, and then:

$$
\frac{D}{C}=\sqrt{3}
$$

This equation relates the amplitude of vibration of atoms $\mathrm{H}_{2}\left(\right.$ or $\left.\mathrm{H}_{3}\right)$ to $\mathrm{N}$ atom; while the motion of Nitrogen atom defined from the molecule center of mass which is kept at constant location ( $\mathrm{z}=0.31426 \AA$ above c-point) through the molecule vibration, i.e. $\mathrm{N}$ atom moves in $-\mathrm{x}$-direction, $\mathrm{x}_{\mathrm{cm}}$ represents the $\mathrm{x}$-component of center of mass, Hydrogen atoms are set in the order illustrated in Fig. (4): 


$$
\begin{gathered}
x_{c m}=\frac{1}{M} \sum_{i} x_{i} m_{i}=\frac{1}{(3 H+N)}\left[(-A+B) H+\left(\frac{A}{2}\right) H+\left(\frac{A}{2}\right) H-C N\right]=0 \\
\frac{B}{C}=\frac{N}{H}
\end{gathered}
$$

And hence;

$$
\frac{B}{D}=\frac{1}{\sqrt{3}} \frac{N}{H}
$$

In ammonia, the $\mathrm{H}_{1}$ atom vibrated with $\mathrm{x}$-component of amplitude 14 times than Nitrogen vibrates, and 8.08 times than other Hydrogen atoms vibrates.

$\mathrm{E}_{2}$ stretching mode is typical to the $\mathrm{E}_{1}$ mode but the atoms $\mathrm{H}_{2}$ and $\mathrm{H}_{3}$ move towards each other to keep $\sigma_{\mathrm{v} 1}$ but do not verify the $\mathrm{C}_{3}$ rotation condition where the angle between $\mathrm{H}_{2} \mathrm{CH}_{3}$ is not $120^{\circ}$.

$$
\begin{aligned}
& \chi_{E 2}\left(Q_{S 1}\right)=\frac{1}{6}\left[S_{1}+\omega_{3} S_{2}+\omega_{3}^{2} S_{3}+S_{1}-S_{2}-S_{3}\right]=\frac{1}{6}\left[2 S_{1}+\left(\omega_{3}-1\right) S_{2}+\left(\omega_{3}^{2}-1\right) S_{3}\right] \\
& =\frac{1}{6}\left[2 S_{1}+\left(-\frac{3}{2}+i \frac{\sqrt{3}}{2}\right) S_{2}+\left(-\frac{3}{2}-i \frac{\sqrt{3}}{2}\right) S_{3}\right]
\end{aligned}
$$

\subsection{Bending Vibration and Atomic Motion}

The bending vibration motion is associated with the change of bond angles and may be classified into two classes; in-plane and out-of-plane motions. In the case where Hydrogen atoms move towards each other in H-plane, the Nitrogen atom is not moved, and bond angles decrease simultaneously (vibration mode $\mathrm{A}_{1}$ ), see Fig. (5).

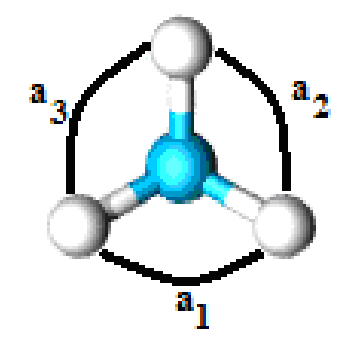

Fig. (5): Bending vibration in Ammonia.

$$
\begin{gathered}
\text { and } \Gamma_{\text {ip }}+\Gamma_{\text {oop }}=\mathrm{A}_{1}+\mathrm{E} \\
\Gamma_{\text {bends }}=\Gamma_{\text {vib }}-\Gamma_{\text {stretches }}=\mathrm{A}_{1}+\mathrm{E}=\mathrm{A}_{1}+\mathrm{E}_{1}+\mathrm{E}_{2}
\end{gathered}
$$




$$
\begin{aligned}
& \chi_{A}\left(Q_{a 1}\right)=\frac{1}{6}\left[a_{1}+a_{3}+a_{2}+a_{1}+a_{2}+a_{3}\right]=\frac{1}{3}\left[a_{1}+a_{2}+a_{3}\right] \\
& \chi_{E 1}\left(Q_{a 1}\right)=\frac{1}{6}\left[a_{1}+\omega_{3}^{2} a_{2}+\omega_{3} a_{3}+a_{1}-a_{2}-a_{3}\right]=\frac{1}{6}\left[2 a_{1}+\left(-\frac{3}{2}-i \frac{\sqrt{3}}{2}\right) a_{2}+\left(-\frac{3}{2}+i \frac{\sqrt{3}}{2}\right) a_{3}\right] \\
& \chi_{E 2}\left(Q_{a 1}\right)=\frac{1}{6}\left[a_{1}+\omega_{3} a_{2}+\omega_{3}^{2} a_{3}+a_{1}-a_{2}-a_{3}\right]=\frac{1}{6}\left[2 a_{1}+\left(-\frac{3}{2}+i \frac{\sqrt{3}}{2}\right) a_{2}+\left(-\frac{3}{2}-i \frac{\sqrt{3}}{2}\right) a_{3}\right]
\end{aligned}
$$

The bending mode $\mathrm{E}_{1}$ just like the $\mathrm{E}_{1}$ stretching mode but the atoms move in H's plane, also $\mathrm{E}_{2}$ mode like the stretching one.

Symmetric state $A_{1}$ : This vibration state is symmetric and the vibration motions are shown in Fig. (6).
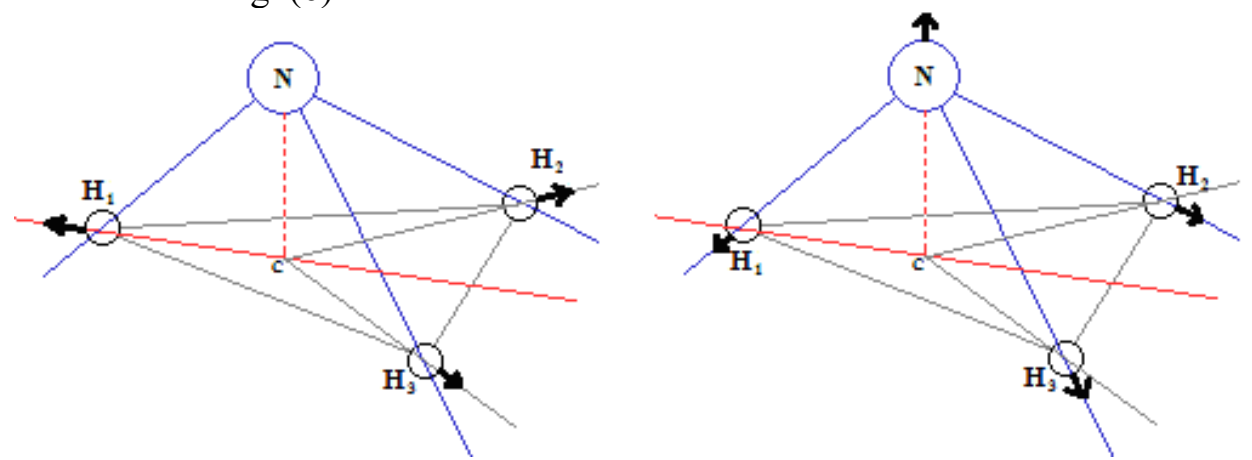

Fig. (6): $A_{1}$ vibration modes in Ammonia.

Degenerate state $E_{1}$ : While this state partially loses the $\mathrm{C}_{3}$ rotation state (Fig. 7), the bond angle $\mathrm{H}_{2} \mathrm{cH}_{3}$ is always $120^{\circ}$, and the bond lengths of $\mathrm{cH}_{2}$ and $\mathrm{cH}_{3}$ are equally in all these vibration motions.

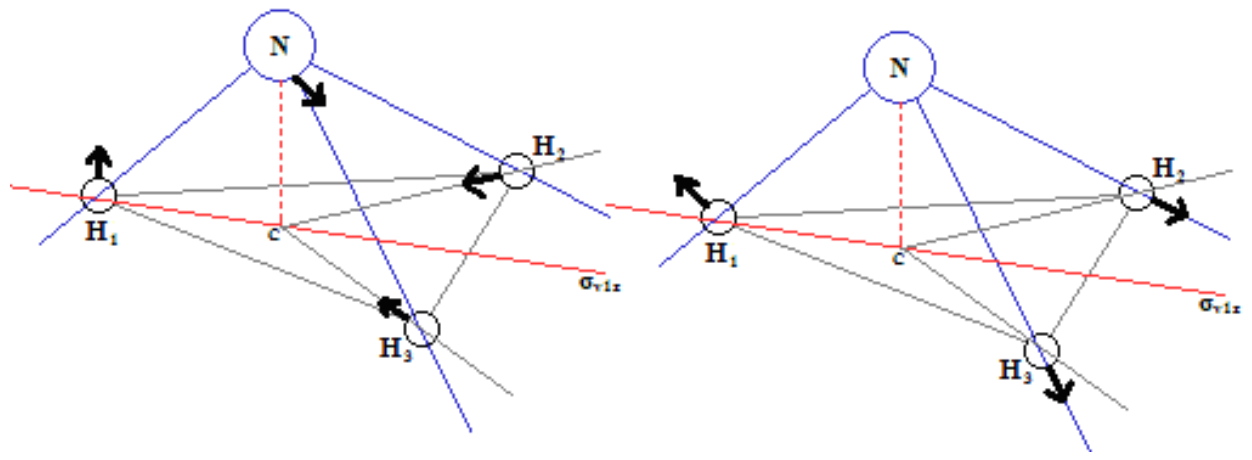

Fig. (7): $E_{1}$ vibration modes in Ammonia.

Degenerate state $E_{2}$ : While this state loses the $\mathrm{C}_{3}$ axis and all vertical mirror plans except $\sigma_{\mathrm{v}}(\mathrm{xz})$. The bond angle $\mathrm{H}_{2} \mathrm{cH}_{3}$ is not saved at $120^{\circ}$ and then $\mathrm{C}_{3}$ rotation lost (Fig. 8). 

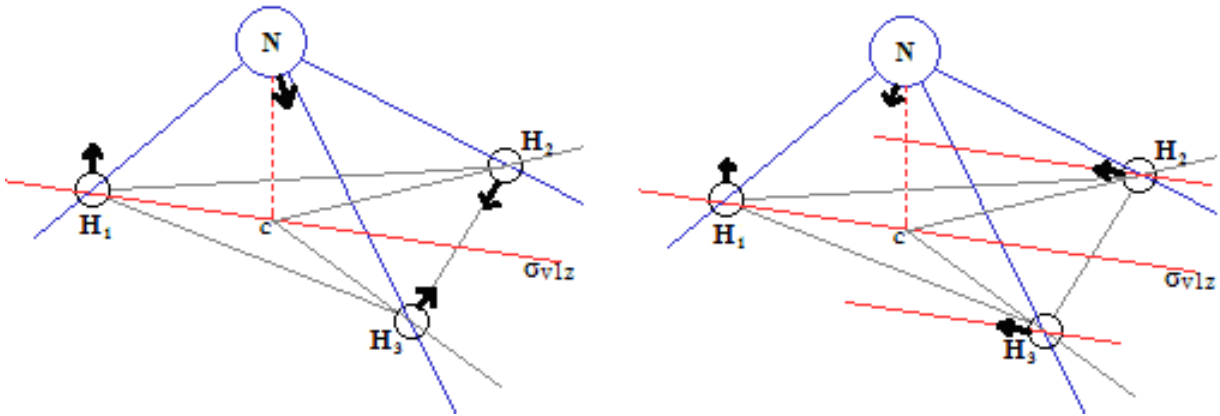

Fig. (8): $E_{2}$ vibration modes in Ammonia.

On the same methods, $\mathrm{C}_{4} / \mathrm{C}_{4 \mathrm{v}}$ point groups can be treated (but requires more restrictions in selection rules) and resulting in the vibration modes shown in Table (6). This point is postponed to future work. The Gray circles represent in-plane motion while signs (+/-) represent out-of-plane in-front/behind the page plane.

Table (6): $\omega$ states for $\mathrm{C}_{4} / \mathrm{C}_{4 \mathrm{v}}$ point groups.

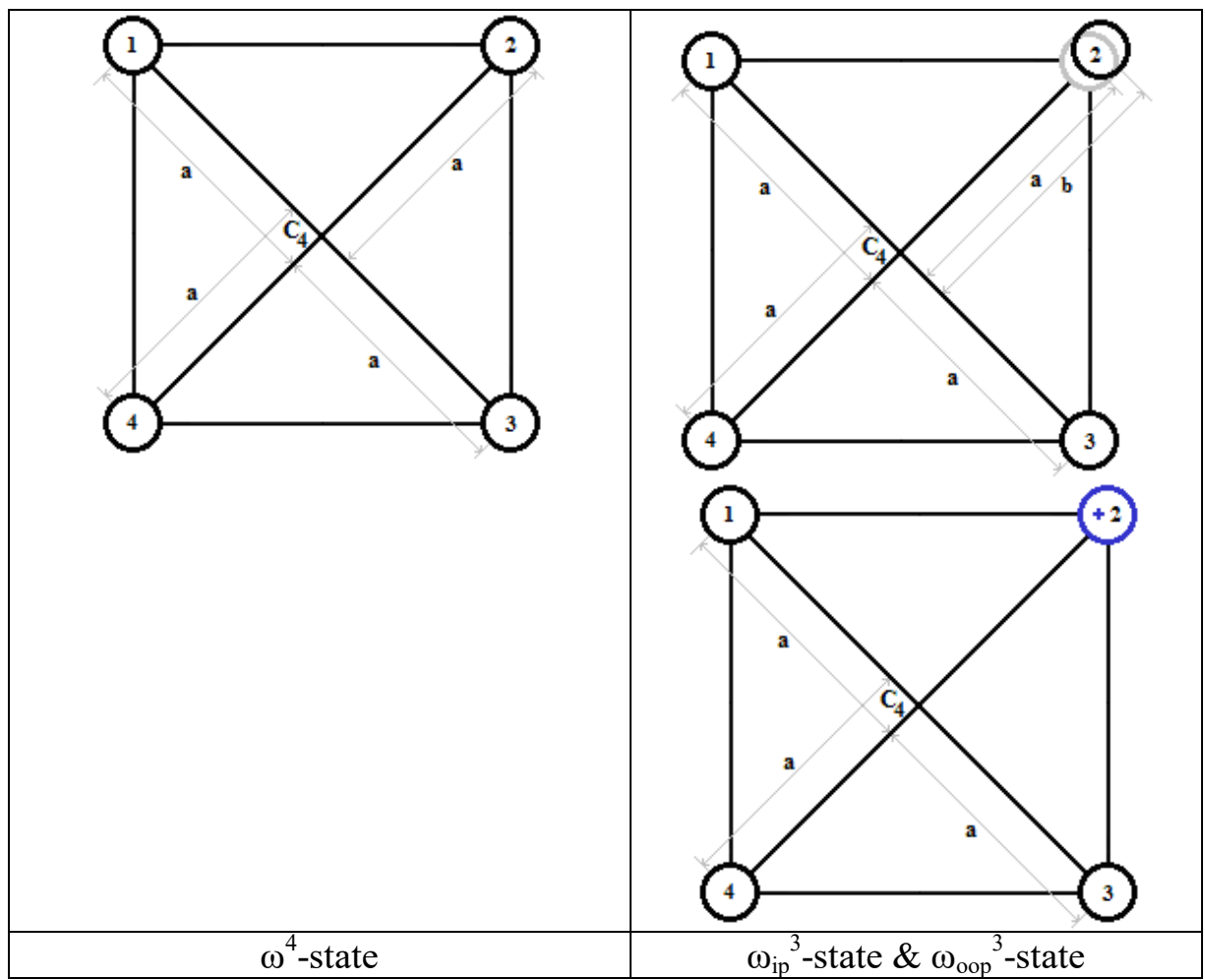




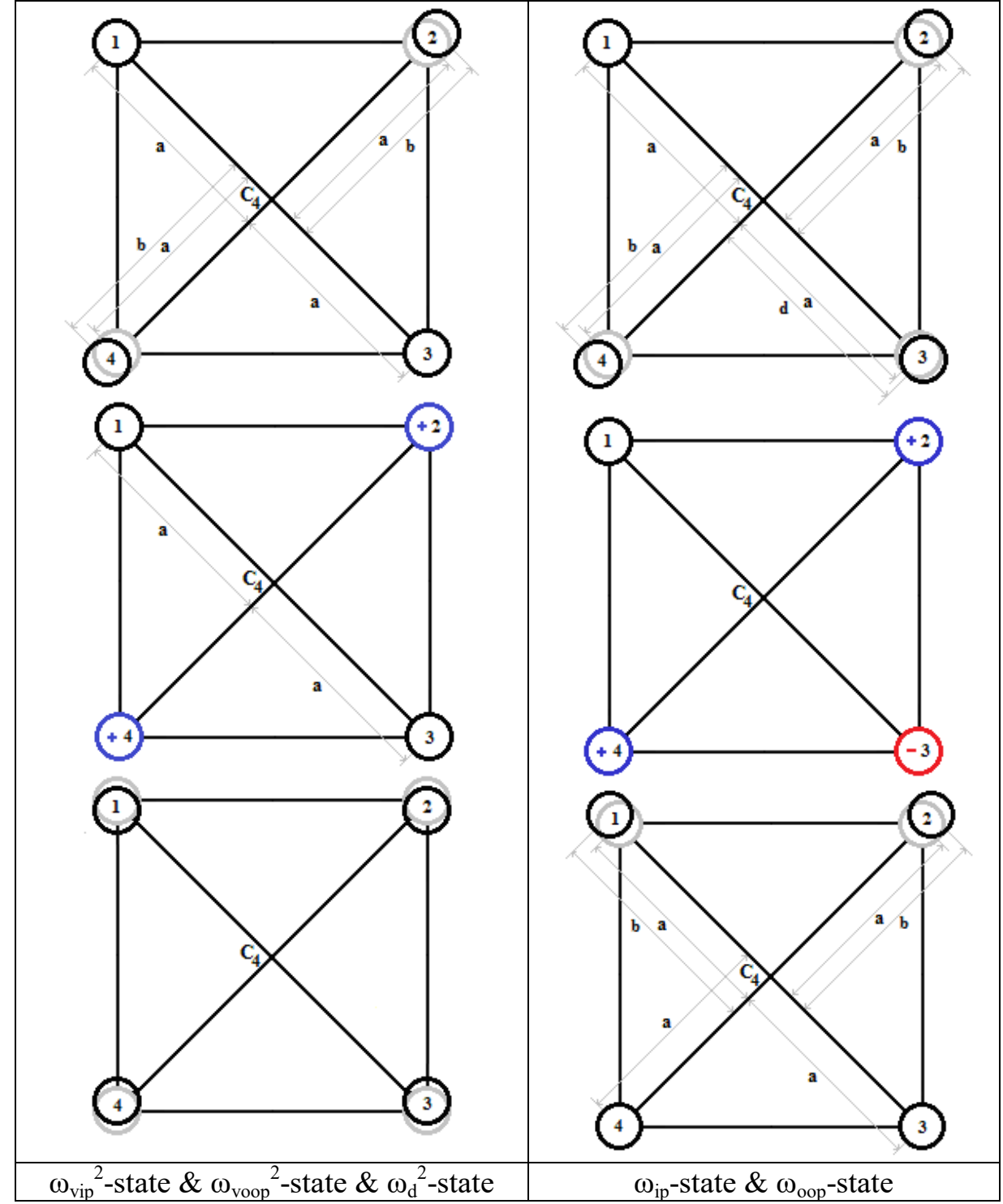

\section{Conclusion}

The concept of partial symmetry is the base of defining the vibrational symmetry states which differs than static one where each symmetry element can possess various levels depending on its order, the building of Omega-table upon the combined vibrational symmetry states and by application of selection rules result in separation the degenerate states and these tables can be reduced to point group character tables. The discussed method of atomic motion shows how molecule partially loses its symmetry but must possess at least very low symmetry value in the lowest symmetry state 
Omega-table describes the atomic motion of the molecule through vibration according to $\omega$ mathematical value and its physical meaning. Then the usage of field theory may discuss the molecular IR and Raman spectra frequency and intensity.

\section{References}

1. M. S. Dresselhaus G. Dresselhaus and A. Jorio. Group theory: Application to the physics of condensed matter (Springer, Berlin, Germany, 2008) CH8.

2. G. Herzberg. Molecular spectra \& molecular structure: II Infrared \& Raman spectra of polyatomic molecules (Von Nostrand Reinhold comp., New York, USA, pp. 462 (1945).

3. P. R. Bunker. Molecular Symmetry and Spectroscopy (Academic Press, Inc., London, New York, 1979) CH7.

4. D. C. Harris and M. D. Bertolucci. Symmetry and Spectroscopy: An Introduction to Vibrational and Electronic Spectroscopy (Courier Corporation, 1978) $\mathrm{CH} 3$.

5. F. Hegelund F. Rasmussen and S. Brodersen. Journal of Raman Spectroscopy 1, 433 (1973).

6. M. A. Perez-Osorio, R. L. Milot, M. R. Filip, J. B. Patel, L. M. Herz, M. B. Johnston and F. Giustino, Journal of Physical Chemistry C119, 25703 (2015).

7. K. Kamaras, L. Badeeb, M. Özeren, A. Pekker, S. Abdel-Aal, A. S. AbdelRahman, P. Andricevic, L. Forro and E. Horvath, Bulletin of the American Physical Society, MARV20013K (2019).

8. S. K. Abdel-Aal, A. S. Abdel-Rahman, W. M. Gamal, M. Abdel-Kader, H. S. Ayoub, A. F. El-Sherif, M. Fawzy, S. Bozhko, E. E. Yakimov and E. B. Yakimov, Acta Crystallographica B75(5) 880 (2019).

9. A. S. Abdel-Rahman and S. K. Abdel-Aal. Molecular Symmetry Degeneracy and Group Theory: Partial molecular symmetry and splitting of degenerate state ISBN 978-3-659-81509-6 (Lambert Academic Publishing, Germany, 2015) $\mathrm{CH} 2$ and $\mathrm{CH} 3$.

10. S. K. Abdel-Aal, A. S. Abdel-Rahman, W. M. Gamal, M. Abdel-Kader, H. S. Ayoub, A. F. El-Sherif, M. Fawzy, S. Bozhko, E. E. Yakimov, E. B. Yakimov, Crystal Structure, Vibrational Spectroscopy, and Optical Properties of $1 D$ Organic-Inorganic Hybrid Perovskite of $\left[\mathrm{NH}_{3} \mathrm{CH}_{2} \mathrm{CH}\left(\mathrm{NH}_{3}\right) \mathrm{CH}_{2}\right] \mathrm{BiCl}_{5}$, Acta Cryst. B75(5) 880 (2019). 